\title{
Improving the results of transarterial embolization of type 2 endoleaks with the embolic polymer Onyx
}

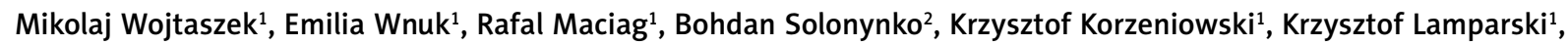 \\ Olgierd Rowinski ${ }^{1}$ \\ ${ }^{1} 2^{\text {nd }}$ Department of Clinical Radiology, Medical University of Warsaw, Warsaw, Poland \\ ${ }^{2}$ Department of General, Vascular and Transplant Surgery, Medical University of Warsaw, Warsaw, Poland
}

Videosurgery Miniinv 2016; 11 (4): 259-267

DOI: https://doi.org/10.5114/wiitm.2016.64747

\begin{abstract}
Introduction: Type 2 endoleaks (T2E) occur in 10 to $20 \%$ of patients after endovascular abdominal aortic aneurysm repair (EVAR) and remain a significant clinical issue.

Aim: To evaluate the efficacy and clinical outcomes of transarterial treatment of persistent type II endoleaks after EVAR using the liquid embolic Onyx.

Material and methods: From February 2012 to August 2015 transarterial T2E embolization was attempted in 22 patients (21 men, median age: 73, range: 62-88 years). Indications for treatment included an increase in the diameter of the aneurysm sac above $5 \mathrm{~mm}$ and a persistent endoleak observed for more than 6 months. Mean time from EVAR to endoleak treatment was 43 months (range: 2-125 months).

Results: Primary technical success was achieved in 17 (77.3\%) patients and secondary technical success in $81.8 \%$, with $0 \%$ in-hospital mortality. The mean procedure time was $95 \pm 48 \mathrm{~min}$, with an average fluoroscopy time of $54 \pm 25 \mathrm{~min}$. The mean amount of Onyx used was $7.5 \pm 6.6 \mathrm{ml}$. Clinical success was seen in 17/21 patients with follow-up imaging (80.9\%). Mean follow-up time was 17 months (range: 3-38 months).

Conclusions: Onyx has been shown to effectively stabilize previous aneurysm growth as a result of the T2E in the majority of our patients. Transarterial embolization of T2E can be significantly improved as compared to previously reported results by using liquid embolic polymers such as Onyx.
\end{abstract}

Key words: endoleak, aortic aneurysm, therapeutic embolization, Onyx copolymer.

\section{Introduction}

A type 2 endoleak, defined as the persistent perfusion of the aneurysm sac, can occur in $10 \%$ to $20 \%$ of patients after endovascular abdominal aortic aneurysm repair (EVAR) and remains a significant clinical issue. Embolization of endoleaks has been performed to date with a variety of embolic agents, namely coils, n-butyl cyanoacrylate (NBCA) glue or thrombin and a number of different approaches, either transarterial, transcaval or with the direct sac puncture technique, with varying results [1-4]. Surgical stent graft explantation also remains a viable treatment option $[5,6]$.

\begin{abstract}
Aim
The aim of this study is to evaluate the efficacy and clinical outcomes of transarterial treatment of persistent type 2 endoleaks (T2E) after EVAR using Onyx, a liquid embolic consisting of a plastic polymer, ethylene vinyl alcohol copolymer dissolved in an organic solvent, dimethyl sulfoxide (DMSO).
\end{abstract}




\section{Material and methods}

\section{Patient population}

Our hospital's investigational review board approved this retrospective study and waived the need for individual patient consent. The review evaluated 22 patients (21 men; median age: 73 , range: $62-88$ ) who underwent transarterial T2E embolization from February 2012 to August 2015 using ethylene vinyl alcohol copolymer as the primary embolization material. Patient demographics, comorbidities, baseline clinical data and risk factors were collected and are reported in Table I. Mean time from EVAR to endoleak treatment was 43 months (range: 2-125 months).

\section{Data collection and image analysis}

Data regarding pre- and postprocedural imaging together with procedure technical details (duration from endograft placement to embolization, access route for embolization, volume of Onyx used), duration of follow-up from embolization and complications were collected based on the review of procedural and imaging reports, angiography and cross-section-

Table I. Patient demographics and comorbidities

\begin{tabular}{|c|c|}
\hline Parameter & Value \\
\hline Age [years] & $73 \pm 9.1$ \\
\hline Male/female & $21 / 1$ \\
\hline \multicolumn{2}{|l|}{ Comorbidities, $n$ (\%): } \\
\hline Cerebrovascular diseases & $6(27.2)$ \\
\hline Neoplasms & $3(13.6)$ \\
\hline Chronic renal failure & $3(13.6)$ \\
\hline Thyroid diseases & $2(9)$ \\
\hline Chronic obstructive pulmonary disease & $1(4)$ \\
\hline \multicolumn{2}{|l|}{ Cardiovascular risk factors, $n(\%)$ : } \\
\hline Hypertension & $14(63)$ \\
\hline Coronary artery disease & $12(54)$ \\
\hline Heart insufficiency & $5(22.7)$ \\
\hline Abnormal heart rhythms & $3(13.6)$ \\
\hline Dyslipidemia & $15(68.1)$ \\
\hline Diabetes mellitus & $5(22.7)$ \\
\hline Smoking habit & $16(72.7)$ \\
\hline
\end{tabular}

al studies and medical records for each patient. The standard imaging protocol at our institution is a single arterial phase computed tomography angiography (CTA) performed at 1, 3, 6 and 12 months with CTA performed yearly afterwards. Ccomputed tomography angiographies were acquired on a 16-row and later on a 64-row multidetector scanner (GE Light Speed 16 or GE Optima 660, GE Medical Systems, Milwaukee, WI, USA) using continuous scans covering the entire aorta, including the proximal supra-aortic vessels down to the groin after intravenous administration of 90$120 \mathrm{ml}$ of non-ionic contrast (Iomeron 370, Bracco Diagnostics, Milan, Italy) at a rate of 3.8 to $4 \mathrm{ml} / \mathrm{s}$ and a bolus tracking threshold of $100 \mathrm{HU}$. A reconstruction increment of $1.25 \mathrm{~mm}$ was used. Images were evaluated independently by two radiologists with 14 (MW) and 4 (EW) years' experience in vascular imaging.

\section{Embolization procedure}

The intention to treat was based on the following indications: evidence of a significant increase in the diameter of the aneurysm sac above $5 \mathrm{~mm}$ with a persistent endoleak observed for more than 6 months in previous cross sectional studies. All patients signed written informed consent prior to the procedure. Technical success was defined as successful filling of the aneurysm sac or, in cases where entry into the endoleak cavity was impossible, permanent occlusion of the feeding vessels on completion angiography. Clinical success was defined as absence of an endoleak with a stable or decreasing aneurysm diameter of aneurysm sac on follow up-imaging. Secondary endpoints included the ability to read with accuracy the postprocedural cross sectional tomographic imaging in relation to the amount of Onyx used and postprocedural complications. The follow-up period was defined as the time from endoleak treatment to the last imaging study on record. All procedures were performed in a dedicated angiography suite (Axiom Artis; Siemens. Erlangen, Germany) with patients in the supine position by a team of interventional radiologists. Vascular access was obtained percutaneously under local anesthesia through the right or left common femoral artery and into the superior mesenteric artery or the hypogastric artery depending on the etiology of the endoleak. Typically, in cases of SMA access, a coaxial system was used with a 6 Fr RDC vascular sheath (Destination, Terumo, Somerset, NJ) inserted into the SMA ostium and a $4 \mathrm{Fr}$ C2 catheter (Glidecath, Terumo, Somerset, NJ) advanced into the 
middle colic artery. After that a 2.4 Fr microcatheter (Rebar, Covidien, Plymouth MN, USA) was advanced through the arc of Riolan into the inferior mesenteric artery (IMA) and the aneurysm sac. A coaxial system similar to that described above was used when cannulating the lumbar arteries with a Simmons 1 or a VS1 catheter (Cook Inc., Bloomington, IN, USA) introduced into the hypogastric artery and a $2.0 \mathrm{Fr}$ microcatheter (Progreat alpha, Terumo, Somerset, NJ) or a 2.4 Fr (Rebar, Covidien, Plymouth MN, USA) advanced into the lumbar artery feeding the endoleak. A similar setting was used when approaching aneurysms of the internal iliac arteries, by cannulating ei-
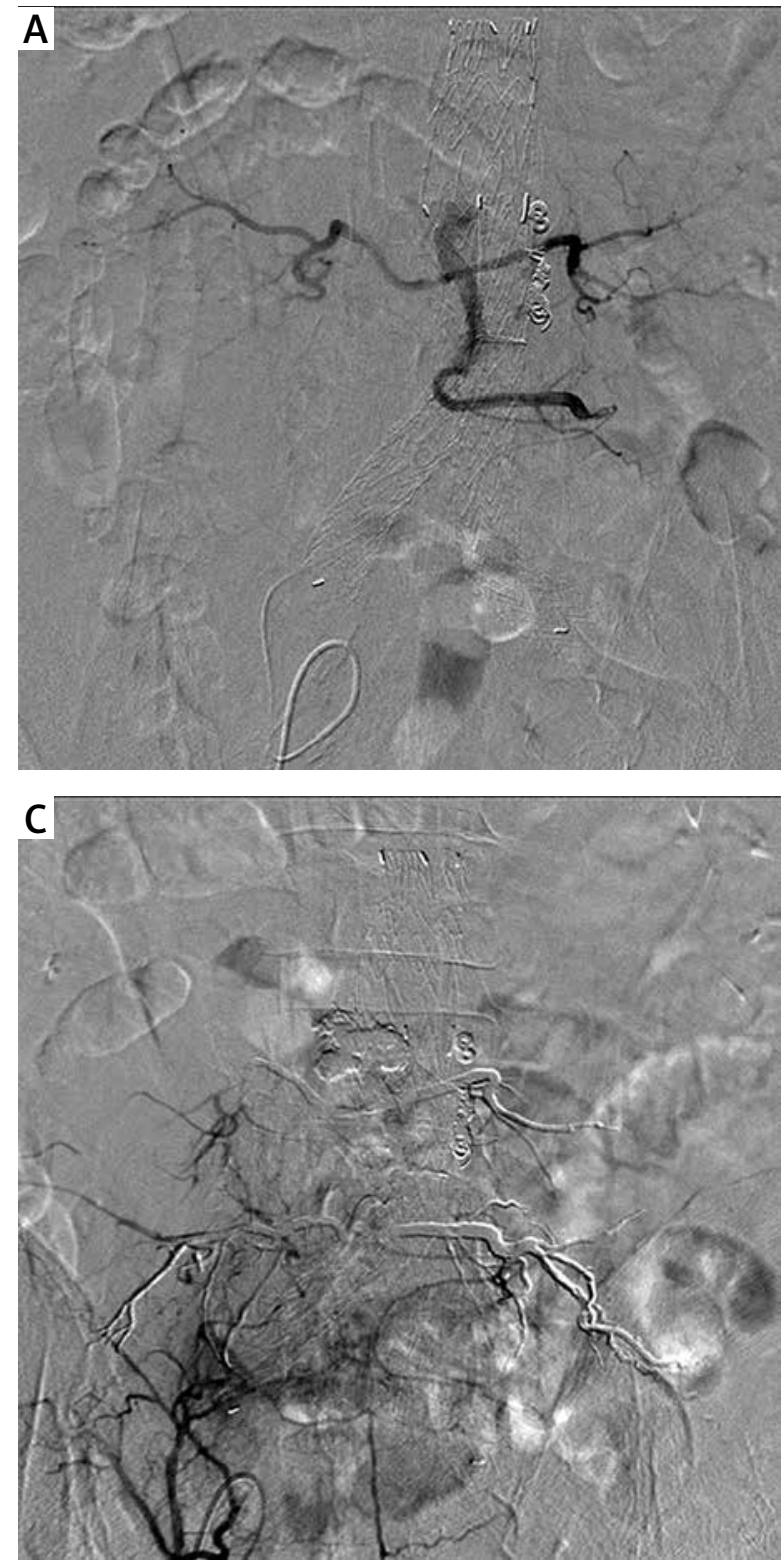

ther the branches of the deep femoral artery or the non-covered contralateral hypogastric artery.

Angiography of the aneurysm sac was performed with assessment of all outflow vessels. Once the fluid dynamics of the endoleak had been evaluated, Onyx was administered into the aneurysm sac under fluoroscopy. Roadmap was used to properly assess the propagation of the embolic fluid. If there was a dominating, high flow outflow route (predominantly drainage into the hypogastric arteries), detachable Concerto microcoils (Covidien, Plymouth MN, USA) were used for its sealing prior to the administration of the embolic polymer (Photo 1). Each

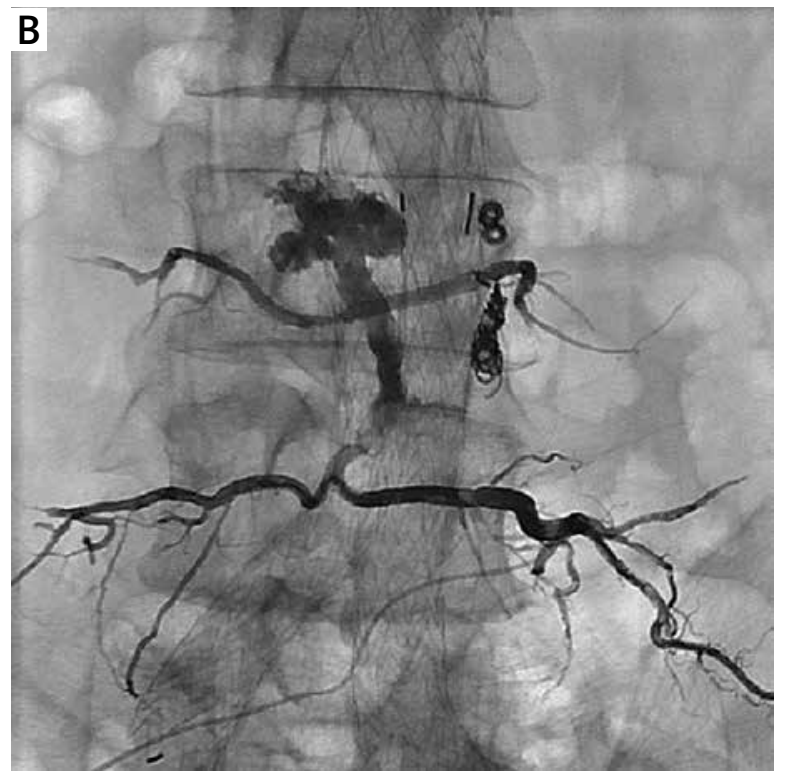

Photo 1. A - Selective catheterization of the hypogastric-lumbar junction feeding an extensive type 2 endoleak supplied/drained by an additional pair of lumbar arteries, $\mathbf{B}$ - since the microcatheter could not be advanced further into the aneurysm sac, the copolymer Onyx was administered "from a distance" and penetrated throughout the endoleak cavity and filled the additional pair of lumbar arteries, C - confirmation angiogram showing successful treatment of the endoleak 
time the intention to treat included full embolization of the endoleak cavity together with the inflow and outflow arteries. When it was impossible to cannulate the aneurysm sac from the hypogastric-lumbar junction, Onyx was administered from a distance, using the more viscous Onyx34 as a plug with an attempt to propagate the lower viscosity Onyx 18 into the endoleak nidus (Photo 2). At the end of the procedure, angiography in several projections was performed to ensure no visible and potentially treatable endoleak remained. In cases where the primary endoleak supply arose from the lumbar arteries a completion angiogram was also performed from
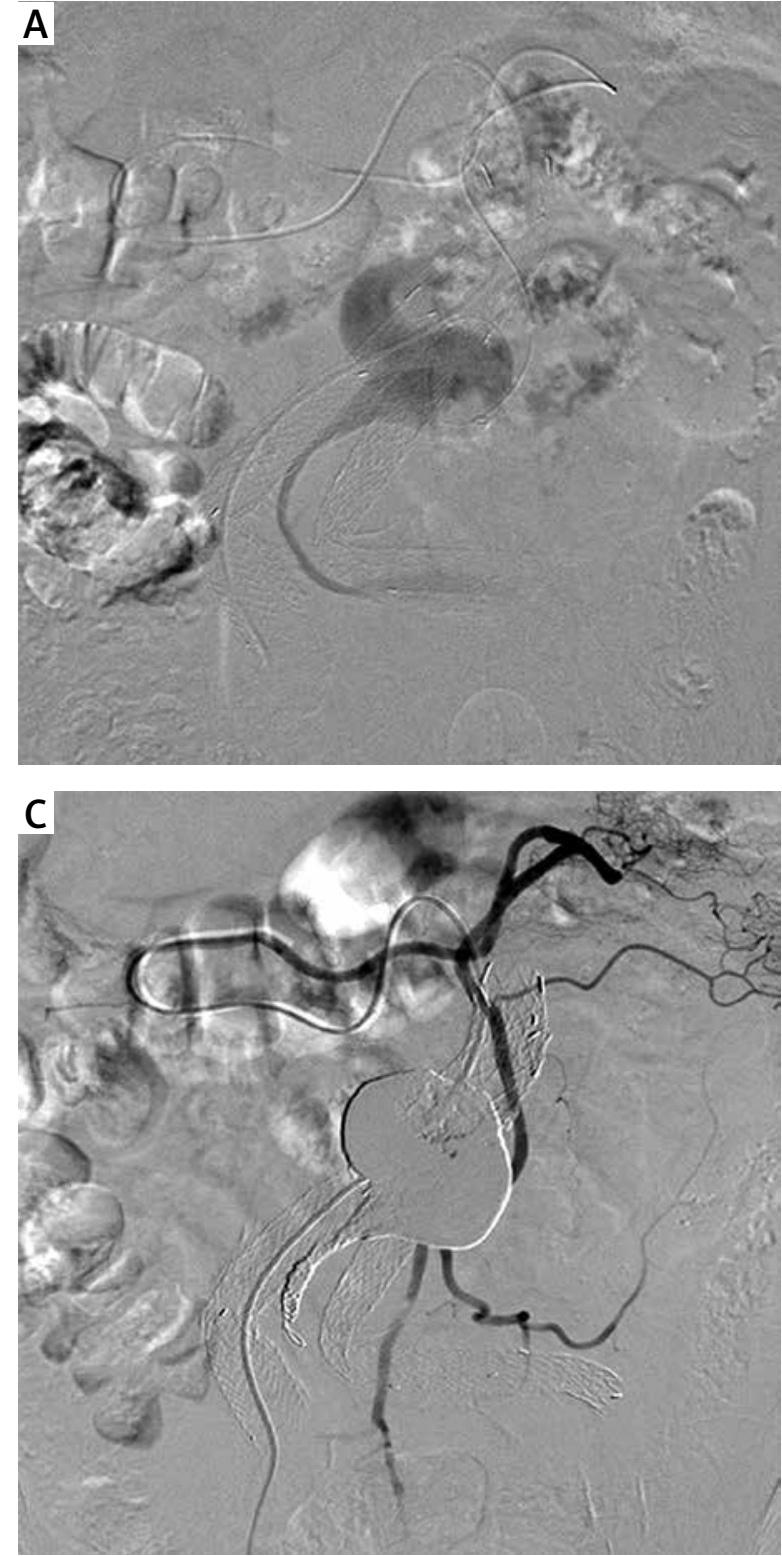

the contralateral hypogastric artery to confirm full exclusion of the endoleak. During follow-up, patients underwent assessment using CTA based on the same preprocedural imaging protocol. Changes in abdominal aortic diameter were assessed and compared to preprocedural imaging in order to evaluate possible sac growth and endoleak recurrence.

\section{Statistical analysis}

All analyses were performed using the method of least squares analysis and correlation tests. Student's $t$ test was used to determine the statistical significance of independent values in least squares

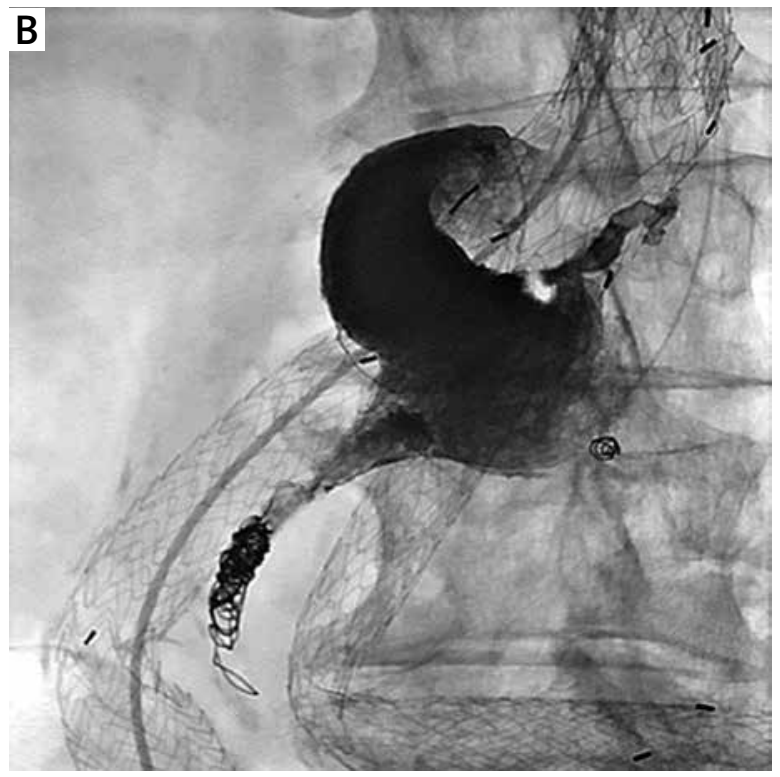

Photo 2. A - Selective catheterization of the SMA-middle colic-IMA collateral pathway (the arc of Riolan) and an angiogram performed showing an extensive endoleak draining into the left hypogastric artery, B - after initial coiling of the outflow vessel, Onyx was administered into the sac filling completely the endoleak cavity, C - confirmation angiogram showing successful treatment of the endoleak together with a fully patent superior rectal artery 
equations, while for correlation tests, the Pearson $r$ correlation matrix was used. A $p$-value $<0.05$ was considered to be statistically significant.

\section{Results}

Transarterial T2E embolization was attempted in 22 patients. Four patients had a previous attempt to seal the endoleak with coiling of the feeding IMA artery through the SMA-middle colic-IMA collateral pathways (arc of Riolan). Three patients were treated for proximal or distal type I endoleaks prior to T2E embolization. Mean aneurysm diameters at first 3-month CTA after EVAR and just prior to endoleak treatment were $65 \pm 12 \mathrm{~mm}$ and $72 \pm 12 \mathrm{~mm}$, respectively.

Primary technical success was achieved in 17 (77.3\%) patients. In the remaining 5 patients, the aneurysm sac could not be reached, but occlusion of the main inflow vessels was considered sufficient. In-hospital mortality was $0 \%$. One patient with a hypogastric aneurysm required retreatment from the contralateral side, giving, together with this staged procedure, a secondary technical success of $81.8 \%$. The mean procedure time was $95 \pm 48 \mathrm{~min}$, with an average fluoroscopy time of $54 \pm 25 \mathrm{~min}$. The mean amount of Onyx used (Onyx34 and Onyx18) was 7.5 $\pm 6.6 \mathrm{ml}$. Complete filling of the aneurysm sac was possible in 17 out of 22 patients. One patient was lost in follow-up, having a brain tumor diagnosed just after the embolization procedure. Apart from this patient, CTA follow-up imaging was available for all the remaining 21 patients, for which the mean follow-up time was 17 months (range: 3-38 months).

Clinical success, described as absence of an endoleak with a stable or decreasing aneurysm diameter, was observed in 17 patients, out of 21 patients with follow-up imaging (80.9\%), with endoleaks reoccurring in 4 patients. Two patients required translumbar (direct sac puncture) embolization for T2E at 25.2 and 37.5 months of follow-up. One patient required an additional iliac branch extension for a type 1 endoleak (T1E) at 24.4 months after T2E embolization and 99.3 months after EVAR. As mentioned before, another patient required a secondary transarterial, contralateral approach to fully treat a hypogastric artery aneurysm. Procedure-specific and follow-up details broken down according to access route are listed in Table II. On follow-up CTA two patients demonstrated a thickening (up to $10 \mathrm{~mm}$ ) of the aortic wall above the embolized sac which was described as similar in appearance to an inflammatory aneurysm (Photo $3 \mathrm{~A}$ ). No sign of infection was present throughout the follow-up period in these patients, nor were the inflammatory response markers elevated. No major complications relating directly to the procedure were experienced in this study population.

The method of least squares was used to analyze the relation between the amount of Onyx and image quality. The latter is represented by separate variables standing for poor, average and good quality of images. Postprocedural CTA images of 5 patients were assessed as poor, 10 as average and 6 as good (Photo $3 \mathrm{~B}-\mathrm{C}$ ). $R^{2}$ statistics proved that $35 \%$ of variability of the dependent variable is explained by independent variables. The results (poor $=14$; average $=-6.6$ and good $=10.66 ; p<0.05$ ) proved that worsening CTA image quality is directly related to the amount of Onyx used.

\section{Discussion}

Type 2 endoleaks remain the Achilles' heel of endovascular repair, with incidence rates up to $20 \%$ [7, 8]. The relevance of T2E is still debatable. Although reports are available showing late rupture in $6 \%$ of patients with persistent T2E with or without aneurysm enlargement, nearly half of endoleaks will resolve spontaneously, with nearly $90 \%$ resolving within the first year after EVAR [9-11]. On the other hand, the persistence of sac pressurization resulting from $\mathrm{T} 2 \mathrm{E}$ leads to the enlargement of the aneurysm sac and degeneration of the aneurysm neck, which may lead to late secondary T1E, placing the patient at higher risk for rupture [12]. When to initiate treatment is still a general unknown, as these statements are unfortunately not statistically supported by a meta-regression analysis published by Karthikesalingam et al., where an optimal threshold for intervention in T2E could not be established [13]. Most authors agree that treatment is warranted in cases of persistent sac perfusion lasting more than 6 months and aneurysm growth of more than $5 \mathrm{~mm}$ per year $[14,15]$. Several methods of endoleak management have been proposed. Approaches include transarterial management and embolization of the inflow and outflow arteries through access by the arc of Riolan or the hypogastric-lumbar junctions, transcaval puncture into the aortic sac or direct translumbar or 
Table II. Intraprocedural and follow-up results for transarterial embolization with data broken down in regards to the access route $(N=22)$

\begin{tabular}{|c|c|c|c|}
\hline \multirow[t]{2}{*}{ Variables } & \multicolumn{3}{|c|}{ Access route for transarterial embolization } \\
\hline & SMA/IMA & Hypogastric/lumbar & Hypogastric/hypogastric \\
\hline N & 9 & 11 & 2 \\
\hline$M / F$ & $9 / 0$ & $10 / 1$ & $2 / 0$ \\
\hline Onyx 34 & $8.7 \pm 9.4$ & $5.6 \pm 4.8$ & $1 \pm 1.4$ \\
\hline Onyx 18 & $0.6 \pm 1.4$ & $1.09 \pm 1.5$ & $2 \pm 1.4$ \\
\hline Onyx 18 and 34 & $9.4 \pm 9.1$ & $6.7 \pm 4.0$ & 3 \\
\hline Additional outflow coil embolization & 3 ( 3,3 and 4 coils) & 0 & 0 \\
\hline Complete sac exclusion & 8 out of 9 & 8 out of 11 & 0 \\
\hline Embolized outflow vessels 1 pair & 2 & 8 & 1 \\
\hline Embolized outflow vessels 2 pair & 0 & 2 & 1 \\
\hline Procedure time [min] & 107 & 92 & 61 \\
\hline Fluoroscopy [min] & 67 & 49 & 37 \\
\hline Technical success & $8 / 9(88.9 \%)$ & 10/11 (90.9\%) & $1 / 2(50 \%)$ \\
\hline $\begin{array}{l}\text { Primary clinical success } \\
\text { (free from endoleak/no sac enlargement) }\end{array}$ & $6 / 9(66.7 \%)$ & $\begin{array}{l}\text { 9/10 (90\%) (1 patient } \\
\text { died before follow-up) }\end{array}$ & $2 / 2(100 \%)$ \\
\hline Subsequent intervention & DSP (2) & - & $\begin{array}{l}\text { Iliac extension for late } \\
\text { T3E (1) } \\
\text { Subsequent contralateral } \\
\text { embolization (1) }\end{array}$ \\
\hline Secondary clinical success & $8 / 9(88.9 \%)$ & - & $2 / 2(100 \%)$ \\
\hline Follow-up, mean (range) [months] & $20(3-38)$ & $16(3-35)$ & $11(8-14)$ \\
\hline
\end{tabular}

SMA/IMA - superior mesenteric artery - inferior mesenteric artery collateral pathway, hypogastric/lumbar - hypogastric - lumbar artery collateral pathway, hypogastric/hypogastric - hypogastric - hypogastric collateral pathway used in the treatment of hypogastric aneurysms, DSP-direct sac puncture (translumbar puncture), T3E - type 3 endoleak.

transabdominal puncture of the aneurysm sac [4, 15]. One benefit of transarterial embolization over translumbar (direct sac puncture - DSP) embolization is that it allows for control angiograms to be performed throughout the embolization process, something that cannot be easily achieved during a translumbar puncture in the prone position most commonly used with a lumbar access. The results of early transarterial embolization have been mostly unsatisfactory [16]. Sarac et al. demonstrated only 44\% freedom from sac expansion using NBCA and coils, while Gallagher KA reported successful embolization after 2 years in $72 \%$ where the IMA was the dominant endoleak source and $17 \%$ for endoleaks supplied by the lumbar arteries $[17,18]$. These unsatisfactory results derive from the nature of NBCA itself. Being a rapidly polymerizing agent, its chance of fully penetrating the nidus before it fully solidifies is limited, leaving spaces in between the embolized endoleak and the thrombus in the aneurysm sac which could provide the grounds for recanalization and reoccurrence of the endoleak. Also, the use of NBCA is hindered by the rapid method in which it has to be delivered in order to fill as much volume of the nidus of possible without "gluing in the catheter". A similar theoretical exercise can be applied to the use of coils during endoleak embolization, used both with NBCA glue and with Onyx. Coils, which provide a scaffolding for fluid embolics, may limit the amount of embolic needed (especially considering the expensive price tag accompanying the use of Onyx), but they will also promote the formation of empty spaces between the coil, the embolic and the aneurysm sac thrombus. Onyx consists of eth- 

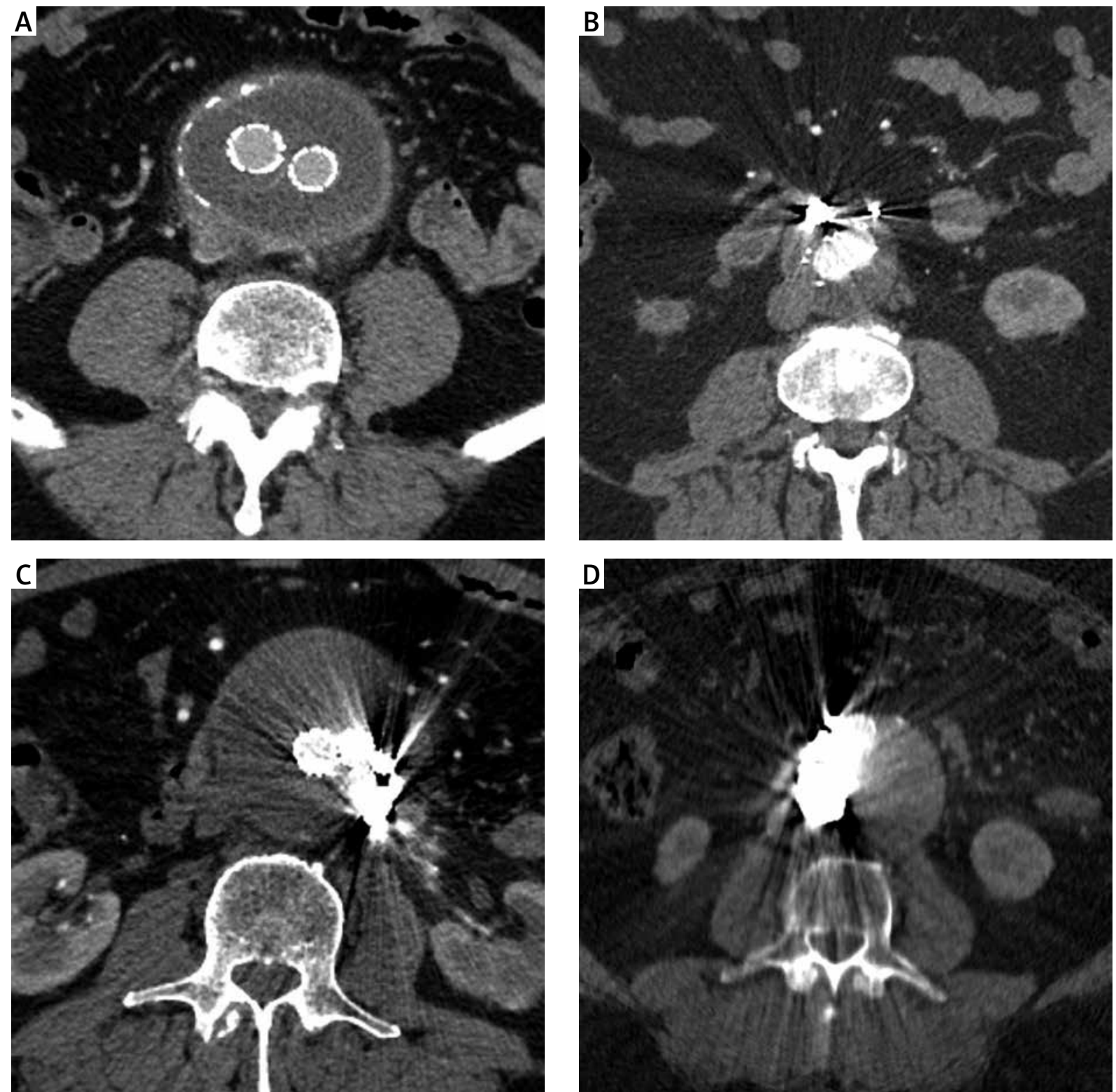

Photo 3. Follow-up CTA imaging. A - Wall thickening observed in two patients after Onyx embolization; Streak artifacts after Onyx embolization in some cases significantly impairing image readability, B - good image quality, C - average image quality, D - poor image quality. Statistical relation was found between the amount of Onyx used and the image quality

ylene vinyl alcohol copolymer (EVOH) dissolved in dimethyl sulfoxide (DMSO), which constitutes 92\% (for Onyx34) and 94\% (for Onyx18) of the injectable formulation. As the applied cast grows during administration into the aneurysm sac, the polymerization rate of Onyx rapidly slows as blood is pushed out from the endoleak, slowing down the elimination and increasing the concentration of DMSO in the cast. This makes the cast even more pliable (as a result of dilution of the $\mathrm{EVOH}$ ) than when administered directly into the bloodstream as during AVM embolization, making filling of the endoleak sac more efficient. Stopping administration of Onyx prematurely on the other hand will theoretically cause the same effect of cavern formation as predicted for the use of fast polymerizing NBCA glue or fluid embolics with coils. Also, it is worth remembering that Onyx has a tendency to form layers when the 
patient is lying in the supine position. We chose to aggressively, continuously and completely fill the sac with the increasingly low viscous Onyx, which was probably the reason why the total amount of Onyx used in our patients was higher than in other reports $(8.25 \mathrm{ml}$, range: $1.5-48 \mathrm{ml})$ available in the current literature. Bosiers et al. reported a mean volume of $4.6 \mathrm{ml}$ of Onyx with a range from 1.1 to $11 \mathrm{ml}$ in $10 \mathrm{pa}$ tients, while Khaja et al. reported a mean volume of $5.6 \mathrm{ml}$ with a range of $2.5-13 \mathrm{ml}$ in 18 patients with a late clinical success rate of $61 \%[19,20]$. One significant disadvantage of Onyx embolization over the use of other embolics is the intense presence of streak artifacts in cross sectional $X$ ray images formed from beam hardening on the suspended tantalum powder. This can significantly impede the readability of follow-up images and hence bias their interpretation in favor of the procedure, which we consider a limitation of the study. In our review of follow-up imaging 15 out of 21 (71.4\%) follow-up images were considered poor or average, with a statistical relationship observed between the amount of Onyx used and the quality of follow-up CTA images. In the future it may be more appropriate to schedule MRA studies in patients who received more than $14 \pm 2.5 \mathrm{ml}$ of Onyx during embolization, as this imaging modality is not hindered by streak artefacts [21]. Also, it is interesting to note that directly after the procedure the aneurysm sac slightly increased in diameter to a mean of $2.98 \mathrm{~mm}$, before stabilizing in consecutive follow-up scans. The nature of this observation is not entirely obvious, but we attribute it to the presence of the polymer which now fills the entirety of the endoleak volume. In the following CTAs further increases in volume were no longer observed, but the initial follow-up CTA may be of some concern for the unexpecting observer.

Another cause for concern is the intraarterial administration of large quantities of DMSO. Dimethyl sulfoxide is an important aprotic solvent that can solubilize a wide variety of otherwise poorly soluble polar and nonpolar molecules. This, coupled with its apparent low toxicity, has led to its ubiquitous use and widespread application, including as the primary solvent for EVOH. Reports have been lately published in which DMSO has been shown to cause toxicity in developing cells through plasma membrane pore formation and induction of apoptosis in doses above $0.3 \mathrm{ml} / \mathrm{kg}[22,23]$. Even though our popula- tion group was above the mean age of 73 , an attempt was made to determine relevant biochemical factors which could be affected from the follow-up test. Unfortunately, the data which were available from our retrospective analysis did not allow us to form any reasonable questions regarding this issue, leaving the topic still open for further assessment. The only complication which occurred in our study group which we directly relate to the embolic polymer was the observed inflammatory thickening of the aortic wall seen on follow-up images in two patients. Although unexpected, this diagnostic finding had no further clinical sequelae.

Our study has the common limitations of retrospective research, including the lack of a defined, randomized comparison group and technical variations in procedure technique. The total number of patients as well as the follow-up period in the series is relatively small, although comparable to other reports.

\section{Conclusions}

Onyx has been shown to effectively stabilize previous aneurysm growth as a result of the type II endoleak in the majority of our patients. Transarterial embolization of type II endoleaks can be significantly improved as compared to previously reported results by using liquid embolic polymers. Still, these have to be followed up closely as treatment of one endoleak does not exclude the chances of developing a late secondary endoleak.

\section{Conflict of interest}

The authors declare no conflict of interest.

\section{References}

1. Sheehan MK, Barbato J, Compton CN, et al. Effectiveness of coiling in the treatment of endoleaks after endovascular repair. J Vasc Surg 2004; 40: 430-4

2. Baum RA, Cope C, Fairman RM, Carpenter JP. Translumbar embolization of type 2 endoleaks after endovascular repair of abdominal aortic aneurysms. J Vasc Interv Radiol 2001; 12: 111-6.

3. Rial R, Serrano FJ, Vega M, et al. Treatment of type II endoleaks after endovascular repair of abdominal aortic aneurysms: translumbar puncture and injection of thrombin into the aneurysm sac. Eur J Vasc Endovasc Surg 2004; 27: 333-5.

4. Scali ST, Vlada A, Chang CK, Beck AW. Transcaval embolization as an alternative technique for the treatment of type II endoleak after endovascular aortic aneurysm repair. J Vasc Surg 2013; 57: 869-74. 
5. White GH, May J, Petrasek P, et al. Endotension: an explanation for continued AAA growth after successful endoluminal repair. J Endovasc Surg 1999; 6: 308-15.

6. Wachal K, Szmyt K, Oszkinis G. Diagnosis and treatment of a patient with type IV endoleak as a late complication after endovascular aneurysm repair. Videosurgery Miniinv 2014; 9: 667-70.

7. Silverberg D, Baril DT, Ellozy SH, et al. An 8-year experience with type II endoleaks: natural history suggests selective intervention is a safe approach. J Vasc Surg 2006; 44: 453-9.

8. United Kingdom EVAR Trial Investigators; Greenhalgh RM, Brown LC, Powell JT, et al. Endovascular versus open repair of abdominal aortic aneurysm. N Eng J Med 2010; 362: 1863-71.

9. Jones JE, Atkins MD, Brewster DC, et al. Persistent type 2 endoleak after endovascular repair of abdominal aortic aneurysm is associated with adverse late outcomes. J Vasc Surg 2007; 46: 1-8.

10. Tolia AJ, Landis R, Lamparello P, et al. Type II endoleaks after endovascular repair of abdominal aortic aneurysms: natural history. Radiology 2005; 235: 683-6.

11. Nolz R, Teufelsbauer H, Asenbaum U, et al. Type II endoleaks after endovascular repair of abdominal aortic aneurysms: fate of the aneurysm sac and neck changes during long-term follow-up. J Endovasc Ther 2012; 19: 193-9.

12. Hansen CJ, Kim B, Aziz I, et al. Late-onset type II endoleaks and the incidence of secondary intervention. Ann Vasc Surg 2004; 18: 26-31.

13. Karthikesalingam A, Holt PJ, Vidal-Diez A, et al. Predicting aortic complications after endovascular aneurysm repair. Br J Surg 2013; 100: 1302-11.

14. Aziz A, Menias CO, Sanchez LA, et al. Outcomes of percutaneous endovascular intervention for type II endoleak with aneurysm expansion. J Vasc Surg 2012; 55: 1263-7.

15. Nevala T, Biancari F, Manninen H, et al. Type II endoleak after endovascular repair of abdominal aortic aneurysm: effectiveness of embolization. Cardiovasc Intervent Radiol 2010; 33 278-84.

16. Baum RA, Carpenter JP, Golden MA, et al. Treatment of type 2 endoleaks after endovascular repair of abdominal aortic aneurysms: comparison of transarterial and translumbar techniques. J Vasc Surg 2002; 35: 23-9.

17. Sarac TP, Gibbons C, Vargas L, et al. Long-term follow-up of type II endoleak embolization reveals the need for close surveillance. J Vasc Surg 2012; 55: 33-40.

18. Gallagher KA, Ravin RA, Meltzer AJ, et al. Midterm outcomes after treatment of type II endoleaks associated with aneurysm sac expansion. J Endovasc Ther 2012; 19: 182-92.

19. Bosiers MJ, Schwindt A, Donas KP, Torsello G. Midterm results of the transarterial use of Onyx in the treatment of persisting type II endoleaks after EVAR. J Cardiovasc Surg (Torino) 2013; 54: 469-75.

20. Khaja MS, Park AW, Swee W, et al. Treatment of type II endoleak using Onyx with long-term imaging follow-up. Cardiovasc Intervent Radiol 2014; 37: 613-22.

21. Nogueira RG, Bayrlee A, Hirsch JA, et al. Dynamic contrast-enhanced MRA at $1.5 \mathrm{~T}$ for detection of arteriovenous shunting before and after Onyx embolization of cerebral arteriovenous malformations. J Neuroimaging 2013; 23: 514-7.
22. Hanslick JL, Lau K, Noguchi KK, et al. Dimethyl sulfoxide (DMSO) produces widespread apoptosis in the developing central nervous system. Neurobiol Dis 2009; 34: 1-10.

23. Galvao J, Davis B, Tilley M, et al. Unexpected low-dose toxicity of the universal solvent DMSO. FASEB J 2014; 28: 1317-30.

Received: 22.06.2016, accepted: 4.12.2016. 